\title{
AN INCREMENTAL METHOD FOR THE CONSTRUCTION OF THE BOX EXTENTS OF A CONTEXT
}

\author{
SÁNDOR RADELECZKI AND LAURA VERES
}

\begin{abstract}
In this paper we are improving a method proposed in [2] for the construction of the box extents of a given formal context. We prove that the lattice of the box extents can be order-embedded in the lattice generated by the atomic extents of the given context.
\end{abstract}

\section{INTRODUCTION}

The notion of the box extents of a context and the box elements of a concept lattice are related to the application of Formal Concept Analysis [1] in data classification by defining partitions of a universe putting its elements with similar attributes into the same class, see $[4,6]$ and [2]. This task transparently appears in some clustering problems originating in database analysis, as well as in the engineering discipline Group Technology, see, e.g., [9] or [8]. This discipline exploits the similarities between technological objects and divides them into (relatively) homogeneous groups in order to optimize the manufacturing processes. In [2] it is proved that the box extents of a formal context form a complete atomistic lattice and two methods for the construction of this lattice are also presented. The second one has an incremental character, it is based on consecutive one-object extensions of a small context. Since, during a one-object extension, the number of box extents can even be doubled, we present a construction and an algorithm to avoid this problem. We also prove that the lattice of box extents can be order-embedded in the lattice generated by the atomic extents of a formal context. These results are presented in Section 3. Section 2 contains some preliminary notions and notations, i.e., the prerequisites of our investigations.

\section{Preliminaries}

For the lattice-theoretic terminology used we refer to [3]. A formal context is a triple $\mathcal{K}=(G, M, I)$, where $G$ and $M$ are sets and $I \subseteq G \times M$ is a binary relation. $G$ is called the object set and $M$ the attribute set of the context $\mathcal{K}$. The basic notions of Formal Concept Analysis can be found, e.g., in [1] or [10]. There

$M S C$ (2010): primary 06B23, 06B05; secondary 06B15.

Keywords: concept lattice, extent partition, box extent, one-object extension. 
are defined

$$
\begin{aligned}
& A^{I}=\{m \in M \mid g I m \text { for all } g \in A\}, \\
& B^{I}=\{g \in G \mid g I m \text { for all } m \in B\}
\end{aligned}
$$

for all subsets $A \subseteq G$ and $B \subseteq M$. We will use the notations $\left(A^{I}\right)^{I}=A^{I I}$ and $\left(B^{I}\right)^{I}=B^{I I}$. For any $g \in G$, we will write $g^{I}$ and $g^{I I}$ instead of $\{g\}^{I}$ and $\{g\}^{I I}$. The obtained maps $A \rightarrow A^{I}, A \subseteq G$ and $B \rightarrow B^{I}, B \subseteq M$ constitute a Galois connection between the power-set lattices $\wp(G)$ and $\wp(M)$, and the maps $A \rightarrow A^{I I}, A \subseteq G$ and $B \rightarrow B^{I I}, B \subseteq M$ are closure operators on $\wp(G)$ and $\wp(M)$, respectively. A formal concept of the context $\mathcal{K}$ is a pair $(A, B) \in \wp(G) \times \wp(M)$ with $A^{I}=B$ and $B^{I}=A$, where the set $A$ is called the extent and $B$ is called the intent of the concept $(A, B)$. It is easy to check that a pair $(A, B) \in \wp(G) \times \wp(M)$ is a concept if and only if $(A, B)=\left(A^{I I}, A^{I}\right)=\left(B^{I}, B^{I I}\right)$. The set of all concepts of the context $(G, M, I)$ is denoted by $\mathcal{L}(G, M, I)$, and $\mathcal{E}(G, M, I)$ stands for the set of all concept extents of $(G, M, I)$. Ordering $\mathcal{L}(G, M, I)$ as follows

$$
\left(A_{1}, B_{1}\right) \leq\left(A_{2}, B_{2}\right) \Leftrightarrow A_{1} \subseteq A_{2} \Leftrightarrow B_{1} \supseteq B_{2},
$$

we obtain a complete lattice, the concept lattice of the context $\mathcal{K}=(G, M, I)$. Similarly, ordering the extents of $\mathcal{K}$ by $\subseteq$, we get a lattice $(\mathcal{E}(G, M, I), \subseteq)$ isomorphic to the concept lattice $\mathcal{L}(G, M, I)$. Now, let us consider the concepts $\gamma(x)=\left(x^{I I}, x^{I}\right)$ for any $x \in G$. It can be easily proved that for any concept $(A, B) \in \mathcal{L}(G, M, I)$, we have in $\mathcal{L}(G, M, I)$

$$
(A, B)=\bigvee\{\gamma(x) \mid x \in A\}
$$

In view of $(2.1)$, any atom in the lattice $\mathcal{L}(G, M, I)$ has the form $\left(x^{I I}, x^{I}\right)$, where $x \in G$. In this case, the extent $x^{I I}$ being also an atom in $\mathcal{E}(G, M, I)$ is called an atomic extent, and $x \in G$ is called an atomic object. If every nonzero element of a lattice $L$ is a join of atoms of $L$, then $L$ is called an atomistic lattice.

An extent partition of a formal context $(G, M, I)$ is a partition of the set $G$, all blocks of which are concept extents. Clearly, the trivial partition $\{G\}$ is always an extent partition. Note that, since the intersection of extents always yields an extent, the common refinements of extent partitions are still extent partitions. Therefore, the extent partitions of $(G, M, I)$ form a complete $\cap$-subsemilattice of the partition lattice of $G$ and, thus, a complete lattice which will be denoted by $\operatorname{Ext}(G, M, I)$. In particular, there is always a finest extent partition of the context denoted by $\pi_{\square}$. In [2], an algorithm for finding $\pi_{\square}$ was provided and it was pointed out that this can be done in $\mathbf{O}\left(|G|^{2}|M|\right)$ steps. The next definition is also from $[2]:$

Definition 2.1. A set $E \subseteq G$ is called a box extent of be the context $\mathcal{K}=$ $(G, M, I)$ if $E$ is a class of some extent partition of $\mathcal{K}$ or $E=\varnothing$ if $\varnothing^{I I}=\varnothing$.

(Note that if $\varnothing^{I I} \neq \varnothing$, then $\{G\}$ is the only extent partition of the context $(G, M, I))$. The set of all box extents of $\mathcal{K}$ is denoted by $\mathcal{B E}(G, M, I)$. Observe that each object $g \in G$ is contained in a smallest box extent denoted by $g^{\square \square}$, which is the class of the finest extent partition $\pi_{\square}$ of the context $(G, M, I)$ containing $g$. 
Hence, according to [2],

$$
E \in \mathcal{E}(G, M, I) \text { is a box extent if and only if } g \in E \Longrightarrow g^{\square \square} \subseteq E .
$$

As a consequence, we get that the intersection of box extents is also a box extent. As $g^{\square \square}$ is a box extent of $(G, M, I)$, the inclusions $\{g\} \subseteq g^{I I} \subseteq g^{\square \square}$ always hold. In [2], it was proved that, ordering the box extents by $\subseteq$, we obtain a complete atomistic lattice having as atoms the classes of the finest extent partition $\pi_{\square}$. Moreover, this lattice $\mathcal{B E}(G, M, I)$ is a complete $\bigcap$-subsemilattice of $\mathcal{E}(G, M, I)$. If $A$ is a box extent, then the concept $\left(A, A^{I}\right)$ is called a box element of the lattice $\mathcal{L}(G, M, I)$, see [4]. The set of the box elements of $(G, M, I)$ is denoted by $\mathcal{B}(G, M, I)$.

\subsection{Box extents of a subcontext}

Let $\mathcal{K}=(G, M, I)$ be a formal context and $H \subseteq G$. It is well-known that, for each extent $E$ of $(G, M, I)$, the restriction $E \cap H$ is an extent of the subcontext $(H, M, I \cap H \times M)$. Similarly, if $E$ is a box extent of $(G, M, I)$, then $E \cap H$ is a box extent of $(H, M, I \cap H \times M)$, according to [2]. In [2] it is also proved that the finite extent partition $\pi_{\square}^{H}$ of $(H, M, I \cap H \times M)$ is a refinement of the restriction of $\pi_{\square}$ to $H$. We say that the context $(G, M, I)$ is a one-object extension of $(H, M, I \cap H \times M)$ if there exists an element $z \in G$ such that $H=G \backslash\{z\}$.

The following well-known lemma will be useful in our proofs.

Lemma 2.2. (i) Any extent $E$ of a finite formal context $(G, M, I)$ contains an atomic object.

(ii) If $A \subseteq H \subseteq G$, then $A^{I I} \cap H$ is an extent of the subcontext $(H, M, I \cap H \times M)$.

Proof. (i) holds since any extent of a finite context contains an atomic extent. As $A^{I I}$ is an extent of $(G, M, I), A^{I I} \cap H$ is an extent of $(H, M, I \cap H \times M)$, hence, (ii) holds.

The next lemma follows from [2], however, to make our paper self-contained, we provide a direct proof of it.

Lemma 2.3. Let $\mathcal{K}=(G, M, I)$ be a context with $\emptyset^{I I}=\emptyset$, and $A \subseteq G$ the nonempty set of its atomic objects. Then, the box extents of the context $(A, M, I \cap$ $A \times M)$ coincide with its extents and $\mathcal{E}(A, M, I \cap A \times M)$ is an atomistic lattice.

Proof. First, observe that, for all $a \in A, a^{I I} \subseteq A$. Indeed, let $a \in A$ and $g \in a^{I I}$. Then, $g^{I I}=a^{I I}$ yields that $g$ is also an atomic object and hence $g \in A$. As $\emptyset$ is an extent, the atomic extents $a^{I I}$ are mutually disjoint. Since $A=\bigcup\left\{a^{I I} \mid a \in A\right\}$, we get that $\left\{a^{I I} \mid a \in A\right\}$ is the least extent partition $\pi_{\square}$ of $(A, M, I \cap A \times M)$, and for this context $a^{I I}$ coincides to $a^{\square \square}$, i.e., to that class of its $\pi_{\square}$ which contains $a$. Now, let $E$ be an arbitrary extent of the context $(A, M, I \cap A \times M)$. Then, $a \in E$ implies that $a^{\square \square}=a^{I I} \subseteq E$ and this means that $E$ is a box extent. The remaining part of the proof is an obvious consequence of the fact that $\mathcal{B E}(A, M, I \cap A \times M)$ is an atomistic lattice. 


\section{One-objeCt EXtensions of A CONTEXT AND the PROPOSED INCREMENTAL METHOD}

The following lemma shows that one-object extension can be used for an inductive construction of the box extents:

Lemma 3.1. Let $\mathcal{K}=(G, M, I)$ be a finite formal context, $B \neq \emptyset$ a box extent of $\mathcal{K}, H \subseteq G$, and $E=B \cap H$. Then, $E$ is a box extent of the subcontext

$$
(H, M, I \cap H \times M)
$$

and it is extended by successive one-object extensions to $B$ in such a way that, after each step, a box extent of the wider subcontext is obtained.

Proof. Since $G$ is finite, it has the form $G=H \cup\left\{z_{1}, \ldots, z_{k}\right\}, z_{1}, \ldots, z_{k} \notin H$. Hence, either $B=E$, or we have $B=E \cup\left\{z_{1}, \ldots, z_{l}\right\}$ with $1 \leq l \leq k$. Set $H_{j}:=H \cup\left\{z_{1}, \ldots, z_{j}\right\}, 1 \leq j \leq l$. Now, the proof is a trivial consequence of the fact that, for each $j \leq l, B \cap H_{j}=E \cup\left\{z_{1}, \ldots, z_{j}\right\}$ is a box extent of $\left(H_{j}, M, I \cap\right.$ $\left.H_{j} \times M\right)$, and it is formed by a one-object extension with $z_{j}$ from the box extent $E \cup\left\{z_{1}, \ldots, z_{j-1}\right\}$.

In this section we use the results of the article [2], where the authors have shown how the box extents of a context are changed by a one-object extension.

Proposition 3.2. ([2, Prop. 4.5]) If $E$ is a box extent of $(G, M, I)$ and $H=$ $G \backslash\{z\}$ for some $z \in G$, then either

(i) $E$ is a box extent of $(H, M, I \cap H \times M)$ with $E \cap z^{\square \square}=\emptyset$ or

(ii) $E \backslash\{z\}$ is a box extent of $(H, M, I \cap H \times M)$.

In view of the above proposition, in case of a one-object extension, any box extent of the wider context $(G, M, I)$ can be derived from a box extent of the subcontext $(H, M, I \cap H \times M)$. The next theorem shows exactly the conditions under which a box extent of the smaller context "gives life" to a box extent of $(G, M, I)$.

Theorem 3.3. ([2, Thm 5.5]) Let $(G, M, I)$ be a context, $E$ a box extent of the subcontext $(H, M, I \cap H \times M)$ with $H=G \backslash\{z\}$. Then

(i) $E$ is a box extent of $(G, M, I)$ if and only if $z^{\square \square} \cap E^{\prime \prime}=\emptyset$;

(ii) $E^{*}=E \cup\{z\}$ is a box extent of $(G, M, I)$ if and only if $z^{\square \square} \backslash\{z\} \subseteq E$ and $(E \cup\{z\})^{\prime \prime}=E \cup\{z\}$.

Observe that, in view of the above theorem, in case of a one-object extension, we have two possibilities:

1) $E$ is also a box extent in the new context iff $z^{\square \square} \cap E^{\prime \prime}=\emptyset$;

2) or $E \cup\{z\}$ is a box extent in the new context iff $z^{\square \square} \backslash\{z\} \subseteq E$ and $(E \cup\{z\})^{\prime \prime}=E \cup\{z\}$.

By applying this theorem, in [2] an algorithm for determining the list of the box extents of $(G, M, I)$ was constructed by using the list of the box extents of the subcontext $(H, M, I \cap H \times M)$. Unfortunately, the theorem shows that, during a one-object extension of the context $(H, M, I \cap H \times M)$, the box extents can be even doubled, for instance, in the case when $z^{\square \square}=\{z\}$. This means that the 
algorithm discussed in [2] can have an exponential time. In order to eliminate this problem, we first prove:

Proposition 3.4. Let $\mathcal{K}=(G, M, I)$ be a finite formal context, $H \subseteq G$ a nonempty subset containing all the atomic objects of $\mathcal{K}, E$ a box extent of the subcontext $(H, M, I \cap H \times M)$, and $z \in G \backslash H$. Set $H_{*}:=H \cup\{z\}$ and $I_{*}=I \cap H_{*} \times M$. Then, the following cases exclude each other.

(1) $E$ is a box extent of $\left(H_{*}, M, I_{*}\right)$;

(2) $E \cup\{z\}$ is a box extent of $\left(H_{*}, M, I_{*}\right)$.

Proof. Assume that the cases (1) and (2) hold simultaneously and denote by $z^{\square \square_{*}}$ the class of the least extent partition of $\mathcal{K}_{*}:=\left(H_{*}, M, I_{*}\right)$ that contains the element $z$. Then, in view of Theorem 3.3, both conditions $z^{\square \square_{*}} \cap E^{I_{*} I_{*}}=\emptyset$ and $z^{\square \square_{*}} \backslash\{z\} \subseteq E$ are satisfied. Hence, we get

$$
z^{\square \square_{*}} \backslash\{z\}=\left(z^{\square \square_{*}} \backslash\{z\}\right) \cap E \subseteq z^{\square \square_{*}} \cap E^{I_{*} I_{*}}=\emptyset .
$$

Since $\{z\} \subseteq z^{I_{*} I_{*}} \subseteq z^{\square \square_{*}}$, we obtain $z^{\square \square_{*}}=z^{I_{*} I_{*}}=\{z\}$. On the other hand, because $\mathcal{K}$ is a finite context, there exists at least one atomic object $a \in G$ with $a^{I I} \subseteq z^{I I}$. Now, $a \in H$, according to our assumption. Thus, we obtain:

$a \in a^{I I} \cap H \subseteq z^{I I} \cap H_{*}=z^{I_{*} I_{*}}=\{z\}$, i.e., $z=a$,

which is a contradiction because $z \notin H$ by our construction.

Another problem, which arises when the box extents of a formal context $\mathcal{K}=$ $(G, M, I)$ are constructed by successive one-element extensions from the box extents of a subcontext $(H, M, I \cap H \times M)$, is the requirement to construct in each step the class $z^{\square \square_{*}}$, i.e., the class of the least extent partition of the extended context $\left(H_{*}, M, I_{*}\right)$ containing the new element $z$. The next proposition shows that $z^{\square \square_{*}}$ can be always replaced by the class $z^{\square \square}$ of the extent partition $\pi_{\square}$ of the whole $\mathcal{K}$.

Proposition 3.5. Let $B$ be a box extent of the context $\mathcal{K}=(G, M, I) H \subseteq G$ a nonempty subset, $B$ a box extent of $\mathcal{K}$, and $z \in G \backslash H$ arbitrary. Set $E:=B \cap H$, and $H_{*}:=H \cup\{z\}, I_{*}:=I \cap H_{*} \times M$. If $z^{\square \square} \cap E^{I_{*} I_{*}} \neq \emptyset$, then $E \cup\{z\}$ is a box extent of the subcontext $\left(H_{*}, M, I_{*}\right)$ and the conditions $\left(z^{\square \square} \cap H_{*}\right) \backslash\{z\} \subseteq E$, $(E \cup\{z\})^{I_{*} I_{*}}=E \cup\{z\}$ hold.

Proof. Clearly, $E$ is a box extent of the subcontext $(H, M, I \cap H \times M)$. Since $E^{I_{*} I_{*}} \subseteq E^{I I} \subseteq B^{I I}=B$, by our assumption, we have $z^{\square \square} \cap B \neq \emptyset$. Since $B$ is a box extent of $\mathcal{K}$, it is a union of some classes of the finest extent partition $\pi_{\square}$ of $\mathcal{K}$ and, hence, $z^{\square \square} \cap B \neq \emptyset$ yields $z \in z^{\square \square} \subseteq B$. Thus, we obtain:

$$
B \cap H_{*}=B \cap(H \cup\{z\})=(B \cap H) \cup\{z\}=E \cup\{z\},
$$

and hence $E \cup\{z\}$ is a box extent of $\left(H_{*}, M, I_{*}\right)$. Then $(E \cup\{z\})^{I_{*} I_{*}}=E \cup\{z\}$ also holds. Now, suppose by contradiction that $\left(z^{\square \square} \cap H_{*}\right) \backslash\{z\} \nsubseteq E$. Then, there is a $g \in z^{\square \square} \cap H_{*}, g \neq z$ with $g \notin E$. However $g \in H_{*} \backslash\{z\}=H$ yields $g \in z^{\square \square} \cap H \subseteq B \cap H=E$, which is a contradiction. Thus, $\left(z^{\square \square} \cap H_{*}\right) \backslash\{z\} \subseteq$ E. 
Remark 3.6. Assume now that conditions of Proposition 3.5 are satisfied, let $\pi_{\square}^{*}$ stand for the finest extent partition of the context $\mathcal{K}\left(H_{*}\right)=\left(H_{*}, M, I_{*}\right)$ and denote the class of the element $z$ in $\pi_{\square}^{*}$ by $z^{\square \square_{*}}$ and its class in $\pi_{\square}$ by $z^{\square \square}$. We show that, during the construction of the box extents of $\mathcal{K}=(G, M, I)$ by successive one-object extensions, $z^{\square \square_{*}}$ can be replaced by $z^{\square \square}$ in checking the condition $z^{\square \square} \cap E^{I_{*} I_{*}}=\emptyset,\left(z^{\square \square} \cap H_{*}\right) \backslash\{z\} \subseteq E,(E \cup\{z\})^{I_{*} I_{*}}=E \cup\{z\}$ (where $\left.E \subseteq H_{*}\right)$, respectively, and this will not alter the list of the box extents obtained.

Indeed, $\pi_{\square}^{*}$ is a refinement of the restriction of $\pi_{\square}$ to $H_{*}$, hence $z^{\square \square_{*}} \subseteq z^{\square \square}$, and $z^{\square \square_{*}} \backslash\{z\} \subseteq\left(z^{\square \square} \cap H_{*}\right) \backslash\{z\}$. Then, $z^{\square \square} \cap E^{I_{*} I_{*}}=\emptyset$ implies $z^{\square \square_{*}} \cap E^{I_{*} I_{*}}=$ $\emptyset$, and in view of Theorem 3.3 this means that $E$ is a box extent of $\mathcal{K}\left(H_{*}\right)$; if $\left(z^{\square \square} \cap H_{*}\right) \backslash\{z\} \subseteq E$, then $z^{\square \square_{*}} \backslash\{z\} \subseteq E$ and by Theorem 3.3 we get that $E \cup\{z\}$ is a box extent of $\mathcal{K}\left(H_{*}\right)$. Hence, all the box extents found belong to $\mathcal{K}\left(H_{*}\right)$. As the successive extensions are ending with $(G, M, I)$, the box extents obtained in the last step are box extents of $(G, M, I)$.

Conversely, let $B$ be a box extent of $\mathcal{K}$, as in Proposition 3.5. Then, $B \cap H=E$ is a box extent of $(H, M, I \cap H \times M)$ and, in view of Lemma 3.1, it can be extended into $B$ by successive one-object extensions. In order to prove that each box extent of $\mathcal{K}$ is found by using our new conditions, assume that neither $E$ nor $E \cup\{z\}$ is decided to be a box extent by checking our conditions for an extension with $z \in G \backslash H$. Then, necessarily, $z^{\square \square} \cap E^{I_{*} I_{*}} \neq \emptyset$. However, in view of Proposition 3.5, $B \cap H=E$ and $z^{\square \square} \cap E^{I_{*} I_{*}} \neq \emptyset$ imply $\left(z^{\square \square} \cap H_{*}\right) \backslash\{z\} \subseteq E$ and $(E \cup\{z\})^{I_{*} I_{*}}=E \cup\{z\}$. The last two relations mean that $E \cup\{z\}$ is recognized as a box extent of the context $\mathcal{K}\left(H_{*}\right)$ by our new method, contrary to the assumption. This contradiction shows that all the box extents of $\mathcal{K}$ are generated by checking the new, modified conditions.

Now, based on Propositions 3.4 and 3.5, and Lemma 3.1, we present an improved method for determining the box extents of a context by successive one-object extensions, eliminating the above-mentioned problems. Its steps are the following:

- First we select atomic objects, i.e., those $g \in G$ from the context $\mathcal{K}=(G, M, I)$ for which $\left(g^{I I}, g^{I}\right)$ is an atom and we store them in a list $H_{0}$.

- We construct the context $\mathcal{K}_{0}:=\left(H_{0}, M, I \cap H_{0} \times M\right)$ and the concept lattice $\mathcal{L}\left(H_{0}, M, I \cap H_{0} \times M\right)$ using some well-known method (see, e.g., [7]). In view of Lemma 2.3, the extent lattice $\mathcal{E}\left(H_{0}, M, I \cap H_{0} \times M\right)$ and its box extent lattice $\mathcal{B E}\left(H_{0}, M, I \cap H_{0} \times M\right)$ are identical. Thereafter, the finest extent partition $\pi_{\square}$ of $\mathcal{K}=(G, M, I)$ is also constructed.

- Now, we add the remaining elements $z_{j} \in G \backslash H_{0}(j=1, \ldots, n)$ one by one to the actual subcontext $\mathcal{K}_{j-1}:=\left(H_{j-1}, M, I \cap H_{j-1} \times M\right)$, determining in each step the box extents of the new context $\mathcal{K}_{j}:=\left(H_{j}, M, I \cap H_{j} \times M\right)$ (where $\left.H_{j}:=H_{j-1} \cup\left\{z_{j}\right\}\right)$ by using the class $z_{j}^{\square}$ of $z_{j}$ in the extent partition $\pi_{\square}$ and the list of the box extents of $\mathcal{K}_{j-1}$.

The last inductive step is carried out by the following algorithm:

Algorithm 3.7. Algorithm for determining the list $L$ of the box extents of $\mathcal{K}$ given the set $H_{0}$ of atomic objects, the list $\mathcal{E}_{0}$ of the box extents of $\mathcal{K}_{0}:=$ $\left(H_{0}, M, I \cap H_{0} \times M\right)$, and the list of the box extents contained in $\pi_{\square}$. 
begin

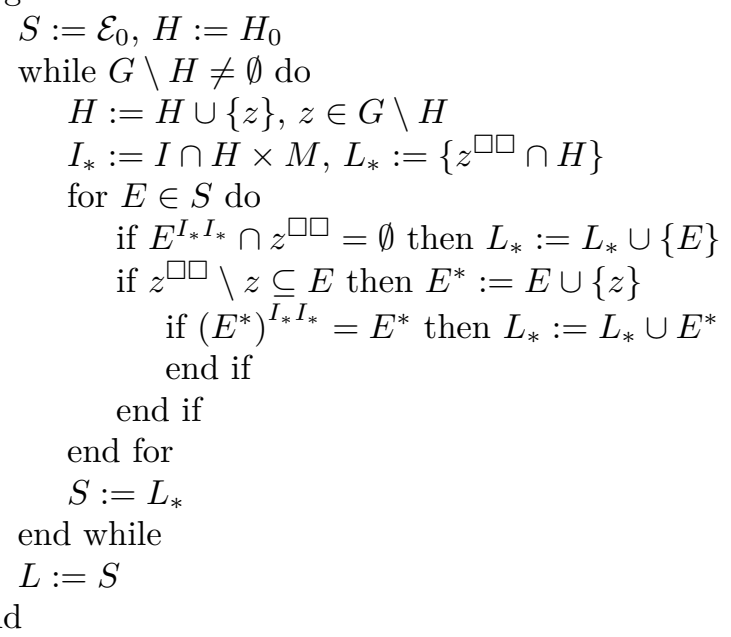

It is known that the lattice $L_{0}:=\mathcal{L}\left(H_{0}, M, I \cap H_{0} \times M\right)$ can be constructed in $\mathbf{O}\left(\left|H_{0}\right|^{2}|M|\left|L_{0}\right|\right)$ steps (see, e.g., [5]) and $\pi_{\square}$ can be computed in $\mathbf{O}\left(|G|^{2}|M|\right.$ ) (see [2]) steps. In the worst case (when every $g \in G$ is an atomic object) $H_{0}=G$ and, hence, $\mathbf{O}\left(\left|H_{0}\right|^{2}\left|M \| L_{0}\right|\right)=\mathbf{O}\left(|G|^{2}\left|M \| L_{0}\right|\right)$. As in each step of the above inductive part at most $\left|L_{0}\right|$ extents are examined, the box extents of the whole context can be checked in at most $\mathbf{O}\left(\left|L_{0} \| G\right|\right)$ steps. Thus, in total, we need at most

$$
\mathbf{O}\left(|G|^{2}\left|M \| L_{0}\right|\right)+\mathbf{O}\left(|G|^{2}|M|\right)+\mathbf{O}\left(\left|L_{0}\right||G|\right)=\mathbf{O}\left(|G|^{2}\left|M \| L_{0}\right|\right)
$$

steps. In the worst case, $\mathbf{O}\left(\left|L_{0}\right|\right)$ is the same as $\mathbf{O}(|\mathcal{L}(G, M, I)|)$.

It is also clear that, in each step, the number of the box extents cannot increase, because a box extent of a subcontext $(H, M, I \cap H \times M)$ is either preserved by a one-object extension or extended with the new element or it disappears and these cases exclude each other according to Proposition 3.4. Therefore, (denoting the set of atomic objects by $A$ ), we obtain

$$
|\mathcal{B E}(G, M, I)| \leq|\mathcal{L}(A, M, I \cap A \times M)| .
$$

The next theorem states even more:

Theorem 3.8. Let $\mathcal{K}=(G, M, I)$ be a finite formal context and $S \subseteq G$ a subset containing all the atomic objects of $\mathcal{K}$. Then, the box extent lattice of $\mathcal{K}$ can be order-embedded in the lattice $\mathcal{B E}(S, M, I \cap S \times M)$.

Proof. Since, in view of [2, Cor. 4.4], for any box extent $E$ of $\mathcal{K}, E \cap S$ is a box extent of the subcontext $\mathcal{K}_{S}:=(S, M, I \cap S \times M)$, we can define the mapping

$$
\varphi: \mathcal{B E}(G, M, I) \rightarrow \mathcal{B E}(S, M, I \cap S \times M), \varphi(E)=E \cap S, E \in \mathcal{B E}(G, M, I) .
$$

Obviously, $\varphi$ is order-preserving. In order to prove that $\varphi$ is an order-embedding, suppose that $\varphi\left(E_{1}\right) \subseteq \varphi\left(E_{2}\right)$ for some $E_{1}, E_{2} \in \mathcal{B E}(G, M, I)$. Then, $E_{1} \cap S \subseteq$ $E_{2} \cap S, E_{1} \cap E_{2} \in \mathcal{B E}(G, M, I)$, and $\varphi\left(E_{1} \cap E_{2}\right) \subseteq \varphi\left(E_{1}\right)$. Assume that $E_{1} \cap E_{2} \neq E_{1}$. This means that there exists an element $z \in E_{1} \backslash\left(E_{1} \cap E_{2}\right)$. Now, let us define the sets $H:=S \cup\left(E_{1} \cap E_{2}\right)$ and $H^{*}:=H \cup\{z\}=S \cup\left(E_{1} \cap E_{2}\right) \cup\{z\}$ and 
consider the corresponding subcontexts $\mathcal{K}_{H}=(H, M, I \cap H \times M)$ and $\mathcal{K}_{H^{*}}=$ $\left(H^{*}, M, I \cap H^{*} \times M\right)$. Clearly, $\mathcal{K}_{H^{*}}$ is obtained from the subcontext $\mathcal{K}_{H}$ by a oneobject extension using the object $z$, and $E_{1} \cap E_{2}=\left(E_{1} \cap E_{2}\right) \cap H=\left(E_{1} \cap E_{2}\right) \cap H^{*}$ is a box extent of both contexts.

On the other hand, $E_{1} \cap S=\left(E_{1} \cap S\right) \cap\left(E_{2} \cap S\right) \subseteq E_{1} \cap E_{2}$ yields

$$
\begin{aligned}
E_{1} \cap H^{*} & =E_{1} \cap\left(S \cup\left(E_{1} \cap E_{2}\right) \cup\{z\}\right)=\left(E_{1} \cap S\right) \cup\left(E_{1} \cap E_{2}\right) \cup\{z\} \\
& =\left(E_{1} \cap E_{2}\right) \cup\{z\},
\end{aligned}
$$

and, hence, $\left(E_{1} \cap E_{2}\right) \cup\{z\}$ is a box extent of $\left(H^{*}, M, I \cap H^{*} \times M\right)$. Therefore, after a one-object extension of the context $\mathcal{K}_{H}$, both $E_{1} \cap E_{2}$ and $\left(E_{1} \cap E_{2}\right) \cup\{z\}$ become box extents in the wider context $\mathcal{K}_{H^{*}}$. Since the set $H \supseteq S$ contains all the atomic objects of the initial context $\mathcal{K}$, this is not possible by Proposition 3.4. This means that $E_{1} \cap E_{2}=E_{1}$ must hold and hence $E_{1} \subseteq E_{2}$. Thus, $\varphi$ is an order-embedding.

\section{REFERENCES}

[1] B. Ganter, R. Wille, Concept Analysis, Mathematical Foundations, Springer Verlag, BerlinHeidelberg-New York, 1999.

[2] B. Ganter, A. Körei and S. Radeleczki, Extent partitions and context extensions, Math. Slovaca 63 (2013), 693-706.

[3] G. Grätzer, General Lattice Theory, Birkhäuser Verlag, Basel-Boston-Berlin, 2003.

[4] A. Körei and S. Radeleczki, Box elements in a concept lattice, in: B. Ganter and L. Kwuida (eds.), Contributions to ICFCA 2006, Verlag Allgemeine Wissenschaft, 2006, pp. 41-56.

[5] L. Kovács, Algorithms for building concept set and concept lattice, Production Systems and Information Engineering 1 (2003), 91-106.

[6] S. Radeleczki, Classification systems and their lattice, Discuss. Math. Gen. Algebra Appl. 22 (2002), 167-181.

[7] S. O. Kuznetsov and S. A. Obiedkov, Algorithms for the construction of concept lattices and their diagram graphs, in: L. De Raedt and A. Siebes (eds.), Principles of Data Mining and Knowledge Discovery, 5th European conference, PKDD 2001, Freiburg, Germany, September 3-5, 2001, Proceedings, Lect. Notes Comput. Sci. 2168, 2001, pp. 289-300.

[8] L. Veres, Classification trees in a box extent lattice, Math. Appl. 17 (2017), 99-110.

[9] T. Tóth, S. Radeleczki, L. Veres and A. Körei, A new mathematical approach to supporting group technology, European J. of Industrial Engineering 8 (2014), 716-737.

[10] R. Wille, Restructuring lattice theory: An approach based on hierarchies of concepts, in: I. Rival (ed.), Ordered Sets, Reidel, Dordrecht-Boston, 1982, pp. 445-470.

Sándor Radeleczki, Institute of Mathematics, University of Miskolc, Address 3515 MiskolcEgyetemváros, Hungary

e-mail: matradi@uni-miskolc.hu

Laura Veres, Institute of Mathematics, University of Miskolc, Address 3515 Miskolc-Egyetemváros, Hungary

e-mail: matlaura@uni-miskolc.hu 